Das Dienstrecht der Vereinten Nationen und der Europäischen Union 


\title{
Die Altersversorgung der internationalen Beamten nach dem Dienstrecht der Vereinten Nationen
}

\author{
Peter A. Köhler
}

I. Die dienstrechtlichen Vorgaben der Charta der VN

II. Das Personalstatut

III. Der Gemeinsame Pensionsfonds

1. Finanzierung

2. Alterspensionen: Anspruchsvoraussetzung und Höhe

3. Besteuerung / besondere Informationspflichten der Organisation

IV. Bewertung 


\section{Die dienstrechtlichen Vorgaben der Charta der VN}

Die Charta der Vereinten Nationen ist beides: Völkerrechtlicher Vertrag ihrer Mitgliedstaaten zur Gründung und Zielbestimmung der Organisation der Vereinten Nationen (,functional sector") einerseits und das für deren Organe verbindliche rechtliche System (,organizational sector $\left.{ }^{6}\right)^{1}$ andererseits.

Nach Art. 101 Abs. 1 und 2 der VN-Charta liegt die Rechtssetzungskompetenz betreffend des Dienstrechts der Bediensteten der Organisation bei der Generalversammlung (General Assembly, GA). Die GA hat neben dem (über die Jahre ständig revidierten) „Personalstatut" ${ }^{2}$ zur Koordinierung des Dienstrechts der im VN-System verbundenen Internationalen Organisationen diverse administrative (Hilfs-) Institutionen errichtet $^{3}$, darunter die für die

,regulation and coordination of the conditions of service of the United Nations common system"4

zuständige International Civil Service Commission, ICSC $^{5}$. Modifikationen des Dienstrechts beschließt die GA seither in der Regel auf Vorarbeit dieser Kommission. ${ }^{6}$

Art. 105 Abs. 2 VN-Charta verleiht den Bediensteten der Organisation ,die Vorrechte und Immunitäten, deren sie bedürfen, um ihre Aufgaben in voller Unabhängigkeit wahrnehmen zu können." Der Generalsekretär der VN7 ist zuständig, im Einklang mit den von der Generalversammlung erlassenen Regelungen das Personal einzustellen. ${ }^{8}$

1 Göttelmann/Münch, Art. 101, A Organizational Power and Authority in Staff Matters, in: Simma u.a., The Charter of the United Nations, A Commentary. 2nd ed. Vol. II, 2002, nr. 2. Zum völkerrechtlichen Aufbau des Organisationensystems s. eingehend Köhler, Sozialpolitische und sozialrechtliche Aktivitäten in den Vereinten Nationen, 1987.

2 Die GA hat die ersten „Staff Regulations“ (Personalstatut) mit GA/Res. 590 (VI) vom 2. Feb. 1952 angenommen, heute gilt mit Wirkung zum 1. Jan. 2003 das mit GA/Res. 57/285 vom 20. Dez. 2002 gebilligte Personalstatut, UN.doc. ST/SGB/2003/5 in der Fassung des ST/SGB/2008/4/1 January 2008.

3 Diese sind aufgeführt bei Göttelmann/Münch, in: Simma u.a., The Charter of the United Nations (Fußn. 1), Art. 101 Rdnr. 19

4 Das „common system“ wurde ursprünglich in den Übereinkommen zwischen den VN und ihren Sonderorganisationen vereinbart, um gemeinsame Standards für die Bediensteten zu entwickeln.

5 Errichtet mit GA/Res 3042 (XXVII) vom 12. Dez. 1972. Zuständigkeit hier zitiert nach deren Statut, Art. 1 Nr. 1, angenommen mit GA/Res. 3357 (XXIX) vom 18. Dez. 1974. Zur Koordination der VN-Administration s. Tassin, Administration Co-ordination in the United Nations Family, in: De Cooker/UNITAR (Eds.), International Administration, Law and Management Practices in Internationale Organisations, 1990, I.2.

6 Göttelmann/Münch, in: Simma u.a., The Charter of the United Nations (Fußn. 1), Art. 101 Rdnr. 23.

7 Bzw. die Generaldirektoren der mit Übereinkommen gem. Art. 57, 63 VN-Charta mit den VN verbundenen Sonderorganisationen für deren Personal.

8 Göttelmann/Münch, in: Simma u.a., The Charter of the United Nations (Fußn. 1), Art. 101 Rdnr. 37. Auf die durch das Budgetrecht der GA faktisch noch weiter limitierte Kompetenz des General- 
Zusätzlich zu den in Art. $100 \mathrm{VN}-$ Charta geforderten Pflichten der Bediensteten - sie dürfen von außerhalb der Organisation „Weisungen weder erbitten noch entgegennehmen“ - legt Art 101 Abs. 3 VN-Charta als ,ausschlaggebenden Gesichtspunkt“ für deren Einstellung prinzipiell ein „Höchstmaß an Leistungsfähigkeit, fachlicher Eignung und Ehrenhaftigkeit" fest.

\section{Das Personalstatut}

Art. 4.2. des Personalstatuts wiederholt für die Ernennung, Versetzung oder Beförderung der Bediensteten die Gesichtspunkte aus Art. 101 Abs. 3 VN-Charta. Die Einstellung erfolgt durch Übergabe eines vom Generalsekretär unterschriebenen „Ernennungsschreibens"9, der Bedienstete gibt „bei der Annahme seiner Ernennung eine Erklärung $\mathrm{ab}$, da $\beta$ er die [im Personalstatut] festgelegten Bedingungen annimmt"“. ${ }^{10}$ Es ist umstritten, ob die Ernennung auf dem Satzungsrecht der Organisation beruht, also selbst Teil des von dieser gesetzten Dienstrechts ist, oder ob sie als Vertrag zwischen zwei gleichberechtigten Partnern zu bewerten ist. Umstritten ist demzufolge auch, ob die Organisation berechtigt ist, das Dienstrecht einseitig abzuändern. ${ }^{11}$ Dabei überwiegt die Meinung, daß das Dienstverhältnis zwar auf Vertrag ${ }^{12}$ beruht, daß aber bestimmte Elemente ,statutory“ 13 sind.

Für Klage aus den „Anstellungsverträgen“ oder „Anstellungsbedingungen“ der Bediensteten ist das Verwaltungsgericht der $\mathrm{VN}^{14}$, United Nations Administrative Tribunal (UNAT) zuständig, für die Arbeitsbedingungen der Bediensteten der ILO das Ver-

sekretärs weisen hin Goodrich/Hambro/Simons, Charter of the United Nations, 3rd ed., 1969, S. 602-603.

9 Personalstatut, Art. 4.1.

10 Ebda., Anhang II, Ernennungsschreiben b).

11 Literatur- und Rechtsprechungsnachweisen s. Göttelmann/Münch, in: Simma u.a., The Charter of the United Nations (Fußn. 1), Art. 101 Rdnr. 78-81; Amerasinghe, Principles of the Institutional Law of International Organizations, 2nd rev. ed., 2005, S. 280-283; Seidl-Hohenveldern/Loibl, Das Recht der Internationalen Organisationen, 1992, Rdnr. 1042, 1045.

12 Seidl-Hohenveldern/Loibl, Das Recht der Internationalen Organisationen (Fußn. 11), Rdnr. 1042, 1045, setzen deshalb das Wort Vertrag in Anführungszeichen, weil sie gleichwohl im folgenden von Beamten sprechen und davon, daß das von der Organisation erlassene Statut durch ,einschlägige gemeinsame Rechtsgrundsätze des öffentlichen Rechts (Beamtenrecht) der Mitgliedstaaten (...) ergänzt wird.“

13 Amerasinghe, Principles of the Institutional Law of International Organizations (Fußn. 11), S. 282: „while the employment relationship is based on contract, there are certain elements which are statutory."

14 Gegründet von der GA mit GA/Res. 351 (IV) vom 24. Nov. 1949. Zur Zuständigkeit s. Art. 2 Ziff. 1 der Resolution. 
waltungsgericht (ILO Administrative Tribunal, ILOAT ${ }^{15}$ ) dieser Sonderorganisation. Beide Gerichte sprechen ,dual nature of the employment relations“. 16 Das UNAT erkannte, daß das Dienstverhältnis auf einem Einstellungsvertrag beruht, dieser aber nur eine Rechtsquelle neben anderen, insbesondere dem jeweiligen Personalstatut ist. ${ }^{17}$ Das ILOAT differenziert im Dienstverhältnis danach, ob die jeweilige Regelung sich auf die Organisation bezieht oder auf die Person des Bediensteten. ${ }^{18}$ Dies führt zu der Feststellung, daß es sich um eine ,special form of employment relationship“ 19 handelt.

Im gleichen Zusammenhang steht Art. 12.1. des Personalstatuts, wonach von der GA das Statut ,unbeschadet der erworbenen Rechte der Bediensteten ergänzt oder geändert werden " kann. Unstreitig ist ${ }^{20}$, daß dadurch eine rückwirkende Verschlechterung der Rechtsstellung der Bediensteten nicht gedeckt wäre. Die Rechtsprechung von UNAT und ILOAT ist darin einhellig, daß eine Statutenänderung nur mit Wirkung für die $\mathrm{Zu}-$ kunft in Betracht kommt und erworbene Rechte nicht tangiert werden dürfen. ${ }^{21}$

In der deutschen Übersetzung des Art. 1.1 a) der „Staff Regulations“ heißt es ohne weitere Differenzierung:

\section{„Die Bediensteten sind internationale Beamte“.22}

Sie haben schriftlich ein feierliches Dienstgelöbnis auf die besonderen Pflichten als internationale Beamte abzulegen, Art. 1.1 b). Diese bestehen neben Loyalitätspflichten

15 Das ILOAT wurde 1947 gegründet. Sein Statut wurde von der Internationalen Arbeitskonferenz 1949 angenommen, es gilt die Fassung vom 11.6.2008. Es ist für die Anstellungsbedingungen der Bediensteten zuständig, nach Art II Nr. 3 ausdrücklich auch ,... to hear any complaint of nonobservance of the Staff Pensions Regulations or of rules made in virtue thereof ..." s. auch Gutteridge, The ILO Administrative Tribunal, in: Cooker/UNITAR (Eds.), International Administration, V.2.

16 Fundstellennachweise bei Göttelmann/Münch, in: Simma, Bruno u.a., The Charter of the United Nations (Fußn. 1), Art. 101 Rdnr. 79.

17 UNAT Judgement No. 19 (1953).

18 ILOAT, Decision No. 61, 12: ,The Staff Regulations and Rules contain in effect two types of provisions, the nature of which differs according to the object, to which they are directed. It is necessary to distinguish, on the one hand provisions which appertain to the structure and functioning of the international civil service and the benefits of an impersonal nature and subject to variation, and, on the other hand, provisions which appertain to the individual terms and conditions of an official, in consideration of which he accepted appointment. Provisions of the first type are statutory in character and may be modified at any time in the interest of the service, subject, nevertheless, to the principle of non-retroactivity and to such limitations as the competent authority itself may place upon its powers to modify them."

19 So Göttelmann/Münch, in: Simma u.a., The Charter of the United Nations (Fußn. 1), Art. 101 Rdnr. 78, ähnlich Amerasinghe, Principles of the Institutional Law of International Organizations (Fußn. 11), S. 282.

20 Literaturnachweise s. dies., a.a.O., Art. 101 Rdnr. 21, und Amerasinghe, a.a.O., S. 306-314.

21 S. etwa ILOAT Judgments nr. 61, nr. 832, nr. 986; UNAT Judgement No. 82, Puvrez (1961), No. 237, Powell (1979) und No. 634, Horlacher (1992) sowie mit weiteren Rechtsprechungsnachweisen Amerasinghe, a.a.O., S. 281.

22 Englisch: „Staff members are international civil servants.“ Im deutschsprachigen Raum hat sich die Bezeichnung ,internationale Beamte” durchgesetzt. 
gegenüber der Organisation und deren Zielen auch aus der Anerkennung von Grundwerten $^{23}$, Verschwiegenheitspflichten (Art. $1.2 \mathrm{~g}$ ) und i), dem Verbot, nationale Orden etc. anzunehmen (Art. $1.2 \mathrm{j}$ ) und einem Genehmigungsvorbehalt für Nebenbeschäftigungen (Art. $1.2 \mathrm{n}$ ). Spezielle Regeln betreffend Form und Fristen für eine Kündigung von Dienstverhältnissen fehlen. Nach Art. 9 kann der Generalsekretär ein Dienstverhältnis beenden, wenn die betreffende Dienststelle wegfällt, dann sind Entschädigungszahlungen vorgesehen, oder wenn der Bedienstete die Ansprüche nach Art. 101 Abs. 3 der VN-Charta nicht erfüllt ${ }^{24}$. Art. 10 ermächtigt den Generalsekretär, eine Disziplinarordnung zu erlassen. Nach Art. 6.1 wird die Mitgliedschaft der Bediensteten im Gemeinsamen Pensionsfonds der VN sichergestellt.

\section{Der Gemeinsame Pensionsfonds}

Die GA beschloß 194925 einen Gemeinsamen Pensionsfonds (UN Joint Staff Pension Fund, UNJSPF), um für die Bediensteten der VN und anderer Internationaler Organisationen Leistungen für das Alter, bei Tod, Invalidität u.a. vorzuhalten. ${ }^{26}$ Die Mitgliedschaft steht den Sonderorganisationen der VN und nach Zustimmung der GA den Internationalen Organisationen offen, die am ,common system“ teilnehmen. ${ }^{27}$ Heute sind das 23 Internationale Organisationen 28 mit zusammen etwa 60.000 Bediensteten. $^{29}$ Der Fonds wird vom UNJSPF-Board, einem ,pension committee“ für jedes Mitglied und einem Sekretariat verwaltet. ${ }^{30}$ Board und ,pension committees" sind ,tripartite“ besetzt, sie werden zu je einem Drittel mit Vertretern der ,participants" (teilnehmende Bedienstete $^{31}$ ), der Mitglieder und des Generalsekretärs beschickt. Der Board hat der GA mindestens zweijährig Bericht zu erstatten. ${ }^{32}$

23 Nach Personalstatut Art. 1. 2. a) sind dies insbes. die in der Charta niedergelegten Grundsätze und die Menschenrechte.

24 Zur Vermeidung von Rechtsstreitigkeiten wird auch hier in der Praxis der „golden handshake“ gewährt, s. Göttelmann/Münch, in: Simma u.a., The Charter of the United Nations (Fußn. 1), Art. 101 Rdnr. 96.

25 GA/Res. 248 (III) vom 23. Jan. 1949.

26 Regulations, Rules and Pension Adjustments System of the UNJSPF, Stand: Jan. 2009, „Scope and Purpose of the Fund".

27 Regulations, Art. 3 (a), (b) und (c).

28 Im Jahr 2008 kam das Special Tribunal for Lebanon dazu, s. UNJSPF, Annual Report 2009, Einführung des Chief Executive Officer.

29 ICSC; General Information, www.icsc.un.org, 1.8.2008.

30 Regulations Art. 4; weitere committees sind für die aktuarische Verwaltung des Fund vorgesehen, s. Art. 9-12.

31 Nach Art. 21 ist jeder Vollzeitbedienstete einer Mitgliedorganisation ,participant“ des Fund. Voraussetzung ist weiter eine medizinische Untersuchung vor Beginn der „participation“, s. ebda., Annex I Administrative Rules of the UNJSPF, sec. C, Medical Examination.

32 Ebda., Art. 14. 


\section{Finanzierung}

Das Fondsvermögen beruht auf den Beiträgen der ,participants“, denen der Mitgliedorganisationen und den aus der Vermögensanlage gezogenen Gewinnen. ${ }^{33}$ In den Worten des Jahresberichts 2009 galt für das Jahr 2008:

„2008 was characterized by unprecedented turbulence in the area of investments.”34

Das Fonds-Vermögen sank von über 40 Milliarden US \$ zum 31. März 2008 auf 31 Milliarden US \$ was einem Verlust von $25 \%$ bedeutet. 35 Dennoch kann der Fonds seinen Verpflichtungen nachkommen,

„In the short-term, contribution income (US \$ 1.84 billion) is almost equal to benefit payments (US \$ 1.87 billion); therefore, the Pension Fund only has to rely on a very small portion (US \$ 38 million) of the income generated from its investment portfolio (US \$ 1.2 billion) to cover its obligations and the costs of its operations. The Pension Fund does not need to sell any securities in order to ensure liquidity." 36

Im Fall, daß das Fondsvermögen nicht ausreicht, um die Verpflichtungen aus den Regulations zu bedienen, sieht Art. 26 eine „Nachschußpflicht“ (,deficiency payments“) der Mitgliedorganisationen proportional zu ihren Beiträgen vor. ${ }^{37} \mathrm{Ob}$ diese Vorschrift den Bediensteten ein subjektives Recht gegen einen Leistungsabbau in der Alterssicherung gibt, war Gegenstand der Rechtsprechung des UNAT: In der Sache Gretz u.a. ${ }^{38}$ ging es um die Anpassung laufender Pensionen an die Preisentwicklung. Die GA entsprach einem Vorschlag des UNJSPF, die zum 1. Januar 1985 fällige Anpassung um 1,5 $\%$ zu kappen mit der Begründung,

„to reduce or eliminate the actuarial imbalance of the Fund ... and thereby to secure an adequate level of benefits for beneficiaries".

Deshalb gilt

,a co-operative effort by member organizations, participants and beneficiaries is required"39.

Die davon betroffenen Antragssteller machten geltend, daß dies ein Fall des Art. 26 sei und beantragten zu entscheiden, daß sie deshalb in ihren Rechten verletzt seien und die Sparmaßnahme aufzuheben sei. Das Gericht verneinte dies:

„IX. In this regard, the Tribunal considers that article 26 does not confer on participants a right directly applicable that can be invoked by the Applicants. There is no basis for the

33 Ebda., Art. 18.

34 UNJSPF, Annual Report 2009 (Fußn. 28), Einführung des CEO.

$35 \mathrm{Zu}$ diesen und den folgenden Zahlen s. UNJSPF, Annual Report 2009 (Fußn. 28), S. 3 ff.

36 So UNJSPF, Annual Report 2009 (Fußn. 28), Bericht CEO.

37 Dazu und zu Finanzierungsproblemen allgemein s. Morgenstern, Social Security Problems, in: Cooker/UNITAR, International Administration (Fußn. 15), IV. 1, insbesondere S. 16 ff.

38 UNAT Judgement No. 403 vom 12. Nov. 1987.

39 GA/Res. 39/246 vom 18 Dez. 1984. 
Applicants' contention that the violation of article 26 would render invalid specific measures aimed at making good a deficiency in the Fund's assets. The validity per se of each such measure must be evaluated by the Tribunal."

Des Weiteren führte es aus, Zuzahlungen der Mitgliedorganisationen nach Art. 26 seien nur eine von vielen Möglichkeiten, den Fonds zu stabilisieren, die aber alle der politisch abwägenden Entscheidung der GA unterliegen. Wenn jedoch Änderungen beim Anpassungsmodus von Pensionen erfolgten, sind bestimmte fundamentale Prinzipien wie

- Rückwirkungsverbot,

- Willkürverbot,

- Zumutbarkeit und die Rationalität der Maßnahme zu beachten. 40

Da die Kappung der Anpassung um 1,5 \% nicht rational begründet wurde, sondern ausschließlich Einsparungen bewirken sollte, hielt UNAT die Maßnahme an der Grenze dessen, was hingenommen werden könnte. ${ }^{41}$

Von daher stand Art. 26 auch einer schrittweisen Erhöhung der Beiträge nicht entgegen. Sie stiegen für die Bediensteten von $7 \%$ des pensionsbegründenden Einkommens im Jahr 1984 auf 7,9 \% seit Januar 1990, die der Mitgliedsorganisationen von 14 \% auf 15,8 \%.42 Zum pensionsbegründenden Einkommen zählt das Grundgehalt nach der im „,common system“ üblichen Berechnungsmethode ${ }^{43}$ sowie Gehaltszulagen für besondere Sprachkenntnisse ${ }^{44}$.

\section{Alterspensionen: Anspruchsvoraussetzung und Höhe}

Das „normale Pensionsalter“ liegt für Geburtsjahrgänge ab dem 1. Januar 1990 bei 62 Jahren. 45 Vorzeitige Pensionierung 46 kommt nach wenigstens fünf Beitragsjahren frühestens ab dem 55. Lebensjahr in Betracht, der Pensionsanspruch wird dabei um $6 \%$ pro Jahr gekürzt. ${ }^{47}$ Ein Aufschub der Pensionszahlungen 48 ist bei vorzeitigem Aus-

40 UNAT Judgement No. 403 unter XI- IV.

41 Ebda., XV: ,such a reduction is on the borderline of what the Tribunal can consider reasonable in the light of its earlier decisions." Es gab zwei abweichende Meinungen, die langfristig wirkende Pensionskürzungen als Verstoß gegen Art. 101 Abs. 3 VN-Charta werteten. Morgenstern, Social Security, S. 25 nennt als eigentlichen Grund, warum Art. 26 nicht angewandt werde, daß letztlich die Mitgliedstaaten der Internationalen Organisationen dies zu finanzieren hätten, was in vielen Fällen nicht durchsetzbar sei.

42 Regulations Art. 25.

43 Ebda., Appendix A, Gross pensionable salary fort he general service and related categories; darin auch die Bezugnahme auf die con der ICSC angenommenen Berechnungsmethode.

44 Ebda., Art. 54 (a) (i) und (ii); zu den Details s. ebda., Anlage B.

45 Ebda., Art. 1, „Normal retirement age“; bis dahin lag die Altersgrenze bei dem 60. Lebensjahr.

46 Ebda., Art. 29 (a).

47 Ebda., Art. 29 (b); unter (i) und (ii) werden Ausnahmen vom Reduzierungsprozentsatz bei Dienstzeiten ab 25 Jahren geregelt. 
scheiden aus dem Dienst möglich. Liegt der Pensionsanspruch bei Erreichen des Pensionsalters unter 1.000 US \$, so kann auf Antrag ${ }^{49}$ des Versicherten eine Einmalzahlung (,lump sum“) geleistet werden. ${ }^{50}$ Wer aus dem Dienst ohne Pensionsanspruch ausscheidet, hat Anspruch auf Leistungen nach dem ,withdrawal settlement", auch hier ist die Wahl einer „lump sum“ möglich. ${ }^{51}$ Dabei werden bei Dienstzeiten unter fünf Jahren die Beiträge zurückgezahlt, bei längerer Dienstzeit wird die Rückzahlungssumme ab dem sechsten Jahr um jährlich $10 \%$ erhöht, höchstens jedoch um $100 \%$.

Die Höhe der Pension wird nach einer komplizierten Formel nach der ,final average remuneration“, dem ,letzten Durchschnittsgehalt", das selbst einer eigenen Formel unterliegt, berechnet: Grundsätzlich werden dafür die 36 vollen Kalendermonate mit dem höchsten pensionsbegründendem Gehalt innerhalb der letzten fünf Beitragsjahre zugrunde gelegt. 52 Von diesem „Endgehalt“" werden nach Zurücklegung der Wartezeit bei Erreichen der Altersgrenze für Dienstverhältnisse ab dem 1. Januar 1983

- für die ersten fünf Beitragsjahre 1,5\%,

- für die nächsten fünf Beitragsjahre 1,75\%,

- für die nächsten 25 Beitragsjahre $2 \%$

- und für über 35 Beitragsjahre ab dem 1. Jul. 1983 1\%, maximal $70 \%$ des „Endgehalts" gezahlt. 53

Geringfügige Pensionen werden in Relation zu den Beitragszahlungen ebenso unter Bezug auf das „UNJSPF Adjustment System“54 angehoben, wie die Mindestpension für Leistungsempfänger ohne weitere Ansprüchen an den UNJSPF. Der Wert laufender Pensionen wird nach diesem Adjustment System dadurch erhalten, daß der US \$ Betrag einmal jeweils dem in den USA errechneten Konsumentenpreisindex angepaßt wird, zum anderen ggfs. dem des Landes, in dem der Berechtigte lebt. ${ }^{55}$

Es besteht die zusätzliche Möglichkeit, Pensionsansprüche ab einer bestimmten Höhe teilweise zu kapitalisieren. Der Betrag der „lump sum“ muß dabei unter 1/3 des aktuarischen Werts der Pension liegen. ${ }^{56}$ Bei Ehepaaren ist ein Anspruch des Partners dann

48 Ebda., Art. 30, Deferred retirement benefit.

49 Ebda., Art. 32 (a).

50 Ebda., Art. 30 (c).

51 Ebda., Art. 31.

52 Ebda., Art. 1 (h); die Vorschrift sieht Abstufungen bei Beitragszeiten unter fünf Jahren vor.

53 Ebda., Art. 28 (a)-(h); für Dienstverhältnisse, die vor 1983 begannen, enthält die Vorschrift Sonderregelungen, für die höchsten Gehaltsstufen ist die Obergrenze bei $60 \%$ des Endgehalts festgesetzt.

54 Dieses wurde gem. GA/Res. 37/131 vom 17. Dez. 1982 den Regulations als Annex III beigefügt.

55 Ebda., Nr. 1-3.

56 Regulations, Art. 28 (g) (i) ,the amount of the lump sum may not exceed the smaller of the actuarial equivalent of one third of the maximum benefit"; er kann also bis zu 32,9\% des Pensionsanspruch betragen. 
ebenfalls zu kapitalisieren. ${ }^{57}$ Minimalpensionen können auf Antrag voll als Einmalzahlung geleistet werden.

Für individuelle Notsituationen von Pensionisten ist ein „Emergency Fund“ vorgesehen, der Hilfen in besonderen Härtefällen bereitstellen soll58. Finanziert aus dem UNJSPF-Vermögen und freiwilligen Zuwendungen, soll „ohne rigides Regelwerk“ ${ }^{\circ 5}$ schnelle, individuell abgestimmte Hilfe ermöglicht werden.

\section{Besteuerung / besondere Informationspflichten der Organisation}

Anders als die laufenden Gehälter, die intern von der Organisation besteuert werden 60 , sind Pensionen allgemein nicht von der nationalen Besteuerung des Landes ausgenommen, in dem der Pensionist lebt. Eine Steuererstattung durch den UNJSPF ist nicht vorgesehen. Bei der Zahlung von „lump sums“ liegt dies insofern anders, als diese als Teil des Endgehalts verstanden werden können und deshalb steuerlich wie diese zu behandeln sind. Dann wäre eine nationale Besteuerung ggfs. durch den Fonds auszugleichen. Dies war auch bis Ende des Jahres 1979 der Fall. Die GA entschied 1979 „endorsing the efforts of the Commission (ICSC) to strengthen the United Nations common system by adjusting it to changing circumstance" 61 , daß diese Praxis zu beenden ist. 62

Diese Entscheidung war Gegenstand diverser Verfahren vor dem UNAT. Hervorzuheben sind die Sache Horlacher ${ }^{63}$ und die Sache Van Leeuwen ${ }^{64}$, weil darin auf eine besondere Fürsorgepflicht der Fondsverwaltung gegenüber den Bediensteten zum Ausdruck kommt:

Der US-Staatsbürger Van Leeuwen wählte die Kapitalisierung von $1 / 3$ seiner UNJSPF-Pension. Da sein Dienstbeginn beim UN High Commissioner for Refugees (UNHCR) 1976 erfolgte, hatte er Anspruch auf Steuererstattung. Er legte den Betrag in

57 Ebda., Art. 28 (g) (iii); die Vorschrift wurde 1955 eingefügt, um die Zahlung sehr kleiner Witwenpensionen zu vermeiden, s. A/2914, Annex II 1955 und UNAT, Judgement Nr. 945, Baldwin (2000).

58 Ebda., Anhang, Note A: UNJSPF Emergency Fund, Ziff. 1.: „It is intended to provide relief in individual cases of proven hardship owing to illness, infirmity or similar cases, including funeral arrangements."

59 Ebda., „without a rigid set of rules“.

60 Personalstatut, 3.3. Assessement.

61 GA/Res 34/165 vom 17. Dez. 1979, Präambel, Satz 3.

62 Im Wortlaut, ebda., unter III: „[The GA] decides that any staff member joining the United Nations Secretariat on or after 1 January 1980 shall not be entitled to receive reimbursement from the Tax Equalization Fund or otherwise for national income taxes paid on lump sum pension payments received from the UNJSPF; this decision will not affect staff members serving with the United Nations prior to 1 January $1980 . "$

63 UNAT Judgement No. 634, Horlacher (1992).

64 UNAT Judgement No. 1185, Van Leeuwen (2004). 
einem „Individual Retirement Arrangement“ (IRA) an, dessen Erträge von den USA besteuert wurden. Im Januar 1995 wurde durch das „Annual Tax Circular” von der für das ,common system" zuständigen Income Tax Unit eine Neuregelung bekannt gegeben ${ }^{65}$, in der es u.a. hieß:

„The United Nations will not reimburse any future taxes payable on such distributions if the income tax liability for these amounts is deferred to future years because the staff member has 'rolled over' the payments into another qualifying pension plan or an IRA account."

Die Steuern aufgrund des „roll over" des Endgehaltsanteils „lump sum“ zu einem IRA-Fund wurde deshalb vom UNJSPF nicht ausgeglichen. Zu entscheiden war primär, ob eine Verschlechterung der Rechtsstellung des Antragstellers per „Steuerrundschreiben“ überhaupt rechtswirksam sei und inwieweit UNJSPF ihn über die steuerlichen Risiken hätte informieren müssen. UNAT entschied, daß der Hinweis, die Steuern auf IRA-Erträge nicht mehr auszugleichen, kein Eingriff in erworbene Rechte des Antragstellers ist, da diese Erträge erst später anfallen und deshalb nicht mit dem „terminal salary" gleichzusetzen sind. Sodann bekräftigt es zwar,

,that ignorance of the law is no excuse, and that each staff member is bound to know the laws which are applicable to him."66

Hier aber sei der Antragsteller sowohl Opfer seines unklugen Handelns, wie auch eines Fehlers der UN-Administration geworden:
„Nevertheless, the Tribunal believes that, in complex matters such as those concerning social security, pensions, taxes or other issues of a similar nature, the Administration has to be especially careful. To this end, the Organization should make every effort to prom- ulgate issuances on these issues, ensuring that they are informative and comprehensive, yet simple and easy to understand. Consideration should be given to the special skills which are necessary for understanding rules of such a technical nature, skills which not everyone possesses. It is especially important that this principle is observed when prom- ulgating rules concerning pension rights, which become relevant for staff members at a period when they are particularly vulnerable." 67

Aufgrund dieser mangelnden Information ${ }^{68}$ wurde dem Antragsteller in Abwägung des beiderseitigen Verschuldens eine einmalige Schadensersatzzahlung in Höhe von US $\$ 25.000$ zugesprochen.

In der Sache Horlacher war der Antragsteller von 1976 bis 1979 Bediensteter der VN, arbeitete dann als freier Berater für die Organisation, um im April 1980 bis zur Pensionierung wieder in diese einzutreten. Als er die Zahlung einer „lump sum“ wählte, wurde der Betrag national besteuert, die VN lehnten eine Steuererstattung ab. Entscheidend war, ob mit dem Wiedereintritt des Antragstellers 1980 der Tatbestand des ,joi-

65 ST/IC/1995/3 of 1 January 1995.

66 Van Leeuwen, unter III., S. 9.

67 Ebda., S. 10.

68 Ebda., unter IV., S. 11: ,appropriate remedy fort he Administration's providing the applicant with the erroneous information, on which he relied to his detriment ". 
ning the United Nations Secretariat on or after 1 January 1980 “ gemäß GA/Res 34/165 vorlag. Einerseits war seine Einstellung 1980 nach den „Staff Rules“69 kein ,reinstatement", sondern eine Neueinstellung im Sinne der Resolution. Andererseits, so trug der Antragsteller vor, war er vor dem Stichdatum Bediensteter der Organisation, so daß er nach dem Wortlaut der Resolution ein Recht auf Steuererstattung hatte. Das Gericht stellte fest, daß die Formulierung in der Resolution

„this decision will not affect staff members serving with the United Nations prior to 1 January 1980."

bezogen auf den Lebenssachverhalt unklar ist. 70 Unter Hinweis auf die eigene Rechtsprechung betr. wohlerworbener Rechte ${ }^{71}$ ermittelte das Gericht den „Willen des Gesetzgebers" dahin, daß die GA primär die Steuererstattung für kapitalisierte Pensionsansprüche beenden, dabei aber die Bediensteten ausnehmen wollte, die am 1. Januar 1980 bereits im Dienst standen. ${ }^{72}$ Deshalb erging Entscheidung dahin, daß dem Antragsteller allein für den Teil der „lump sum“, dessen Anwartschaften er vor 1980 erworben hatte, Steuererstattung zu gewähren ist.

\section{Bewertung}

Selbst wenn man für die Bediensteten im VN-System ein Dienstverhältnis sui generis annimmt, wird doch zu Recht von internationalen Beamten gesprochen. Dies folgt aus den dienstrechtlichen Vorgaben der VN-Charta, aus dem daraus abgeleiteten Sonderstatus der Bediensteten und ihren damit verbundenen besonderen Pflichten, sowie daraus, $\mathrm{da} ß$ das gesamte Dienstrecht auf Statut beruht. Die Pensionsordnung sieht eine leistungsbezogene Pension vor, die sich an dem Gehalt orientiert, das während der letzten fünf Jahre vor Eintritt in den Ruhestand bezogen worden ist. Dabei besteht die Möglichkeit, sich geringfügige Pensionsansprüche ganz, höhere bis zu 1/3 ihres aktuarischen Werts auszahlen zu lassen. Obwohl die Pensionsordnung vorsieht, daß die Mitgliedsorganisationen Finanzierungsprobleme des Gemeinsamen Pensionsfonds auszugleichen haben, wurde diese Vorschrift bislang nicht angewandt. Deshalb waren Reaktionen auf wachsende Risiken des Fonds erforderlich. Diese führten nicht zu einer grundlegenden Reform des Alterssicherungssystems, sondern bestanden aus mehreren moderaten

69 ST/SGB/2002/1. 1 January 2002, Staff Regulations of the United Nations and Staff Rules 100.1 to 112.8, (Consolidated Text), Rule 103. 4: Re-employment. (a) A former staff member who is reemployed shall be given a new appointment or, if re-employed within twelve months of separation from service or a longer period following retirement or disability under the Joint Staff Pension Fund Regulations, he or she may be reinstated in accordance with paragraph (c) below.

70 Horlacher, unter IV, S. 8: ,the language of the last clause of the resolution, relied upon the Applicant, is unclear."

71 Ebda., unter VI., S. 9: Judgement No. 82, Puvrez (1961), No. 237, Powell (1979).

72 Ebda., unter XI, S. 11-12. 
Schritten: Der Anhebung der Altersgrenze auf 62 Jahre, der Verlangsamung des prozentualen Erreichens des höchsten Pensionssatzes und der Anpassung laufender Pensionen, der Erhöhung der Beiträge der Bediensteten um 0,9 \%, der der Mitgliedsorganisationen um 1,8\% und dem Abbau der Steuererstattung bei Kapitalisierung der Pension. Bemerkenswert ist, daß die Rechtsprechung des UNAT notwendige Anpassungen der Pensionsordnung zwar über die Jahre akzeptiert, im Hinblick auf wohlerworbene Rechte der Bediensteten dafür aber enge Grenzen gezogen hat. Zugleich werden hohe Anforderungen an die Fürsorgepflicht des Dienstherrn gestellt, bei Leistungsänderungen mit negativer Auswirkung auf die Bediensteten alle Informationen bereitzustellen, um diese Nachteile möglichst gering zu halten. 


\title{
Die Altersversorgung der Beamten der Europäischen Union
}

\author{
Matthias Knecht
}

I. Grundlegung

1. Die Struktur des öffentlichen Dienstes der Europäischen Union

2. Entwicklung und Reformen des Beamtenrechts der Europäischen Union

3. Die rechtliche Stellung der Beamten der Europäischen Union
a) Beamtenbegriff
b) Voraussetzungen für die Ernennung
c) Rechte und Pflichten
d) Beamtenbesoldung

II. Ausgestaltung der Altersabsicherung von Beamten

1. Ruhestandsalter und Ruhegehalt

2. Die Höhe des Ruhegehalts

3. Ruhegehalt bei Weiter- oder Wiedereintritt in den Dienst

4. Weitere Reformpläne in der Beamtenversorgung

5. Die Finanzierung der Beamtenversorgung

6. Besteuerung der Dienstbezüge und der Beamtenversorgung

III. Auswertung 